\title{
On the Response of Interleaved Transformer Windings to Surge Voltages
}

\section{Pedersen, A.}

Published in:

IEEE Transactions on Power Systems

Link to article, DOI:

10.1109/TPAS.1963.291363

Publication date:

1963

Document Version

Publisher's PDF, also known as Version of record

Link back to DTU Orbit

Citation (APA):

Pedersen, A. (1963). On the Response of Interleaved Transformer Windings to Surge Voltages. IEEE Transactions on Power Systems, 82(66), 349-356. https://doi.org/10.1109/TPAS.1963.291363

\section{General rights}

Copyright and moral rights for the publications made accessible in the public portal are retained by the authors and/or other copyright owners and it is a condition of accessing publications that users recognise and abide by the legal requirements associated with these rights.

- Users may download and print one copy of any publication from the public portal for the purpose of private study or research.

- You may not further distribute the material or use it for any profit-making activity or commercial gain

- You may freely distribute the URL identifying the publication in the public portal

If you believe that this document breaches copyright please contact us providing details, and we will remove access to the work immediately and investigate your claim 
$\cos \left[\left(N_{2}+N_{1}\right) \chi-N_{2} n t\right]$, the ratio of traveling wave and rotor speeds is given by:

$\frac{\text { Speed of permeance wave }}{\text { Rotor speed }}=\frac{N_{2}}{N_{2}+N_{1}}$

If this harmonic permeance wave is to lock in with the fundamental $\mathrm{mmf}$ wave at $3,600 \mathrm{rpm}$, the rotor speed should be:

$$
\begin{aligned}
\text { Rotor speed } & =\frac{N_{2}+N_{1}}{N_{2}} \times 3,600 \\
& =\frac{24+22}{22} \times 3,600=7,527 \mathrm{rpm}
\end{aligned}
$$

It is obvious that this high operating speed has academic interest only. As mentioned by Mr. Koch, external power would probably have to be supplied over the rotor shaft to maintain that rotor speed.

Equation 38 includes only fundamental terms of slot waves. In calculating motor torque, two components should be included. The main component of torque comes from the interaction between the 2-pole permeance wave and the 2 -pole $\mathrm{mmf}$ wave. The other component of torque comes from the interaction between the 46-pole permeance wave (the third term of equation 38 ) and the $23 \mathrm{rd}$ harmonic of the mmf wave. At a rotor speed of $327 \mathrm{rpm}$ backward, the speed of the 46-pole permeance wave for the sample motor is
Speed of 46-pole permeance wave

$$
=\frac{N_{2}}{N_{2}+N_{1}} \times(-327)=-156 \mathrm{rpm}
$$

and

Speed of $23 \mathrm{rd} \mathrm{mmf}$ harmonic

$$
=\frac{-3,600}{23}=-156 \mathrm{rpm}
$$

Therefore, these two waves will react and produce useful reluctance torque.

\section{REFERENCE}

1. The Nature of Polyphase Induction Machines (book), P. L. Alger. John Wiley \& Sons, Inc., New York, N. Y., 1951.

\section{On the Response of Interleaved Transformer Windings to Surge Voltages}

\section{A. PEDERSEN}

\begin{abstract}
Summary: The high series capacitance theory for the response of interleaved trans former windings to surge voltages is criticized from the point of view that an increased series capacitance as a result of interleaving is incompatible with the concept of a pure capacitive initial voltage distribution. A new theory is proposed according to which the distributed earth capacitances are charged up during an initial period by heavy currents flowing into the winding through surge impedances formed by the coils as a result of interleaving. Formulas are derived for the initial voltage distribution and for the maximum axial voltage gradient.
\end{abstract}

COR A TRANSFORMER winding with uniformly distributed earth capacitance $C$ and series capacitance $K$, and the neutral directly grounded, the initial electrostatic voltage distribution set up instantaneously by a rectangular wave of amplitude $E$ is given by

$e(x)=E \frac{\sinh (\alpha x)}{\sinh (\alpha)}$

where $\alpha=\sqrt{C / K}$ and $x$ is the ratio between the axial distance from the neutral and the axial length of the winding, and $e(x)$ is the voltage to earth at the point

Paper 63-17, recommended by the AIEE Transformers Committee and approved by the AIEE Technical Operations Department for presentation at the IEEE Winter General Meeting, New York N. Y., January 27-February 1, 1963. Manuscript submitted April 30, 1962; made available for printing October 22, 1962.

A. Pedersen is with the Technical University of Denmark, Lyngby, Denmark. $x$. Maximum initial voltage gradient occurs at the line end of the winding and is approximately $\alpha$ times greater than it would be for a uniform distribution. For a conventional cylindrical disk-type winding the value of $\alpha$ normally lies between 10 and 20 . A low $\alpha$ value requires a low earth capacitance or a high series capacitance, the latter being the only feasible possibility for a disk-type winding. The interleaved disk winding, which is the subject of this paper, is one attempt at achieving this. However, it will be shown that the improved surge characteristic of this type of winding can be explained without recourse to the hypothesis of an increased series capacitance.

\section{The Interleaved Winding}

In the interleaved disk winding two consecutive electrical turns are separated physically by a turn which is electrically much further along the winding. Fig. 1 (A) shows a cross section of a doublesection interleaved coil. It is wound as a conventional double-section disk coil, but with two wires in parallel. The wires are transposed at the inside and the appropriate wires joined together at the outside, thus forming a single-circuit double-section coil. Each electrical turn may consist of two or more wires in parallel, as shown in Fig. 1(B).

The interleaved disk-type winding was introduced in two papers ${ }^{1,2}$ by Chadwick, Ferguson, Ryder, and Stearn in 1950.
No quantitative theory was given. It was merely assumed that a large increase in the effective series capacitance was obtained by interleaving the turns. It was stated that interleaving of the turns of a double section coil 5 feet in diameter containing 24 turns increased the series capacitance about 30 times. Later, the principle of interleaving was discussed in papers by Grimmer and Teague, ${ }^{3}$ Ferrari Bardile, ${ }^{4}$ Brechna, ${ }^{5}$ Price, ${ }^{6}$ Zambardino, ${ }^{7}$ Gorio, ${ }^{8}$ and Jayaram. ${ }^{9}$

\section{The High Series Capacitance Theory}

Formulas for the calculation of the effective series capacitance of interleaved windings have been proposed in references 5,8 , and 9 . They define the series capacitance as the capacitance which, when charged to the same voltage as the coil, stores an electrostatic energy equal to that stored in the interturn capacitances. It is further assumed that the voltage will be distributed uniformly between the turns of each coil. These assumptions lead to the following approximate formula
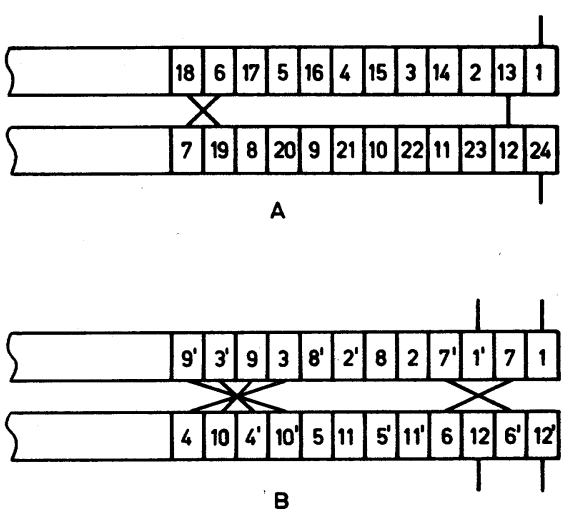

Fig. 1. Section through an interleaved doublesection coil

A. $-n=24, p=1$

B- $n=12, p=2$ 
for the series capacitance $K$ of an interleaved winding.

$K=\frac{(n p-2) c_{t}}{4 N}$

in which $p$ is the number of parallel paths, $n$ the number of electrical turns per doublesection coil, $c_{t}$ the capacitance between two physically adjacent turns, and $N$ the number of series-connected double-section coils. This equation leads to series capacitances which are considerably higher than they would be for a similar noninterleaved winding. The parameter $\alpha$ becomes

$\alpha=\sqrt{\frac{4}{\pi} \cdot \frac{1}{\epsilon \epsilon_{0}} \cdot \frac{N}{n p-2} \cdot \frac{1}{D} \cdot \frac{d}{b} \cdot C}$

in which $\epsilon$ is the relative dielectric constant of the interturn insulation, $\epsilon_{0}$ the permittivity of free space, $8.854 \times 10^{-12}$ farads per meter, $D$ the mean diameter of the coil, $d$ the thickness of the interturn insulation, $b$ the axial width of the copper wire, and $C$ the earth capacitance of the winding. Price ${ }^{6}$ states that $\alpha$ lies between 1 and 3 for a normal interleaved winding.

\section{Analysis of the High Series Capacitance Theory}

All the evidence in favor of the high series capacitance theory is indirect in the sense that it is not based on any direct experimental determination of the series capacitance. What is known is that interleaving improves the response of the winding to surge voltages, and that this apparently can be accounted for by assuming that interleaving increases the series capacitance.

It is inherent in the concept of a pure electrostatic initial voltage distribution that initially no current is allowed to flow through the inductances. It is, therefore, difficult to see that interleaving can have any effect on the equivalent capacitive network. The pure capacitive network of two disk-type windings of exactly the same dimensions, one interleaved and the other noninterleaved, must be identical. Therefore any difference between two such windings can only be accounted for if currents are allowed to flow through the electrical turns of the coils. However, in that case the physical processes cannot be described mathematically by the formulas of the pure capacitance network theory. The idea that the interleaving connections between the two disks of an interleaved double section coil should have any effect upon the capacitive network of the coil cannot be valid. There is no principal differ- ence between such connections and the connections between any two consecutive electrical turns within a coil.

An attempt at assessing experimentally the equivalent series capacitance of an interleaved winding is described by Gorio. $^{8}$ It is based on measurements of the frequency of the free oscillations in a winding which has been removed from the core in order to make the earth capacitance as small as possible. This frequency depends on the inductance and the self-capacitance, which Gorio assumes is identical to the series capacitance. If a known capacitance is connected across the winding, the frequency is reduced and the self-capacitance can be evaluated. Gorio found that the results agreed within $10 \%$ with those predicted from the high series capacitance hypothesis, and this is not surprising. The equivalent capacitive circuit which determines the free oscillation of a winding is by definition very closely linked with the distributed inductances, and the effect of interleaving must necessarily be very pronounced. This is, however, an entirely different situation from that which arises instantaneously when a steep impulse wave is applied to a winding and the electrostatic voltage distribution is set up with no currents flowing in the inductances. The high self-capacitance of an interleaved winding explains the pronounced smoothing effect of interleaving upon the transients between initial and final voltage distribution.

\section{The Initial Voltage Distribution in Interleaved Windings}

Each of the two disks of an interleaved double-section coil forms, when the interleaving joints are disconnected, a spiral-wound parallel plate capacitor with the capacitance

$C=\frac{1}{2}(n p-2) \epsilon_{0} \pi D \frac{b}{d}$

The disk will, however, only behave like a capacitor if the voltage applied to the terminals varies so slowly that the finite charging time of the spiral electrodes can be ignored. When this is not the case the disk will respond like a transmission line characterized by a surge impedance $Z$ and a transit time $T$, which we will define as twice the time taken for a signal to travel thorugh the line. $Z$ and $T$ are given by

$$
\begin{aligned}
& Z=\sqrt{\frac{\bar{L}}{C}} \\
& T=2 \sqrt{ } \overline{L C}
\end{aligned}
$$

where $C$ is the capacitance of the line and $L$ its self-inductance given by

$L=\frac{1}{4} \mu_{o} \pi D \frac{d}{b} \cdot \frac{n}{p}$

And we obtain for $Z$ and $T$

$$
\begin{aligned}
& Z=\mu_{0} c \cdot \frac{d}{b} \cdot \frac{1}{p} \sqrt{\frac{1}{2 \epsilon\left(1-\frac{2}{n p}\right)}} \\
& T=\frac{\pi n D}{c} \sqrt{\frac{\epsilon}{2}\left(1-\frac{2}{n p}\right)}
\end{aligned}
$$

in which $\mu_{0}$ is the permeability of free space, $4 \pi \times 10^{-7}$ henrys per meter, and $c$ is the velocity of light in free space, $3 \times 10^{8}$ meters per second.

Let a rectangular wave of amplitude $E$ be applied across an interleaved doublesection coil at the time $t=0$. The coil may be visualized, apart from the inside crossover, as two series-connected surge impedances of the type previously described. The effect of the inside crossover is not felt at the outside terminals until $t=T$ and the current flowing into the coil will be constant during this interval. At $t=1 / 2 T$ the two surges of amplitude $1 / 2 E$ penetrating the two disks will meet at the inside crossover. By considering the electric fields set up in the interturn insulation by the flow of electric charges along the copper wires it is seen that the meeting of the two waves at $t=$ $1 / 2 T$ results in a doubling of the interturn voltage to $E$ in the form of waves moving back to the outside terminals. At $t=T$ these waves have reached the outside terminals and are reflected back into the disks reducing the interturn voltage to $1 / 2 E$, i.e., the coil will oscillate with the frequency $f=1 / T$. These oscillations will be superimposed upon the voltages induced by the magnetic field linked with the inductive current which will start flowing through the electrical turns. Due to the losses the current will finally become a direct current determined by the total resistance. We may thus divide the whole sequence into three periods: the initial period from $t=0$ to $t=T$ where the coil behaves as two series-connected surge impedances, followed by a transition period in which an inductive current flows through the turns, and the final period where the current is a constant direct current.

A transformer winding consisting of $N$ series-connected double-section interleaved coils will initially respond to a rectangular wave like $2 N$ series-connected surge impedances. Neglecting the time (a few millimicroseconds) it takes the current to travel through all the joints between the coils at the outside of the coil stack, a surge current flowing into the 


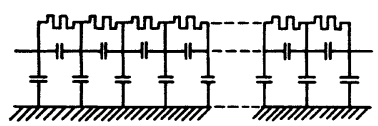

Fig. 2. Equivalent diagram of an interleaved winding for the initial voltage distribution

winding at the line terminal would, but for the effect of the distributed capacitances, immediately appear at the neutral with the same magnitude, and the voltage distribution along the coil stack would be uniform. However, the earth capacitances will be charged up and the distribution becomes nonuniform.

\section{Calculation of the Voltage \\ Distribution During the Initial Period in an Interleaved Winding}

The equivalent diagram of an interleaved winding will, as far as the initial period is concerned, be an $R C$ (resistance capacitance) network of the type shown in Fig. 2. For a wave front of finite steepness we may neglect the effect of the series capacitances which normally represent much higher impedances than the series-connected surge impedances. For a large number of coils the voltage $e(x, t)$ to earth is given by the partial differential equation

$\frac{\partial^{2} e(x, t)}{\partial x^{2}}=R C \frac{\partial e(x, t)}{\partial t}$

in which $x$ is the relative axial distance along the coil stack from the neutral, $C$ is the total capacitance to earth, and $R=$ $2 N Z$ is the resultant surge impedance of the $N$ series-connected double-section interleaved coils. All voltages are assumed to be zero for $t<0$. At $t=0$ a voltage $e(1, t)$ is applied to the line terminal at $x=1$. The neutral at $x=0$ is directly grounded. Let $E(x, s)$ be the Laplace transform of $e(x, t)$ defined by

$E(x, s)=\int_{0}^{\infty} \exp (-s t) e(x, t) d t$

The partial differential equation can then be transformed into the ordinary differential equation

$\frac{d^{2} E(x, s)}{d x^{2}}=R C s E(x, s)$

which, with the given boundary conditions, has the solution

$E(x, s)=E(1, s) \frac{\sinh (\sqrt{R C s} x)}{\sinh (\sqrt{R C s})}$

where $E(1, s)$ is the Laplace transform of the applied voltage. This equation can be expanded in the following way:

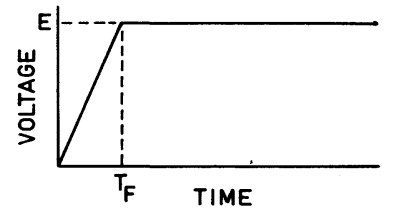

Fig. 3. Idealized surge wave with finite front time

$$
\begin{aligned}
& E(x, s)=E(1, s) \times \\
& \sum_{\nu=0}^{\infty}\{\exp [-\sqrt{R C s}(2 \nu+1-x)]- \\
& \quad \exp [-\sqrt{R C s}(2 \nu+1+x)]\}
\end{aligned}
$$

We assume for simplicity that the front of the applied wave is linear and of a duration $T_{F}$ which is smaller than, or equal to, the duration $T$ of the initial period. The wave tail is infinite and of constant voltage $E$; see Fig. 3 . This wave is given analytically by

$e(1, t)=\frac{E}{T_{F}} t-\frac{E}{T_{F}}\left(t-T_{F}\right) H\left(t-T_{F}\right), \quad t \geqq 0$

in which $H\left(t-T_{F}\right)$ is Heaviside's unit function

$H\left(t-T_{F}\right)=0$ for $t<T_{F}$

$H\left(t-T_{F}\right)=1$ for $t>T_{F}$

The Laplace transform of $e(1, t)$ is

$E(1, s)=\frac{E}{T_{F}} \cdot \frac{1}{s^{2}}\left[1-\exp \left(-T_{F} s\right)\right]$

The inverse Laplace transform of equation 14 then becomes ${ }^{10}$

$$
\begin{aligned}
& e(x, t)=4 E \frac{t}{T_{F}} \times \\
& \sum_{\nu=0}^{\infty}\left\{i i e r f c\left[\sqrt{\frac{R C}{4 t}}(2 \nu+1-x)\right]-\right. \\
& \left.\operatorname{iierfc}\left[\sqrt{\frac{R C}{4 t}}(2 \nu+1+x)\right]\right\}- \\
& 4 E \frac{t-T_{F}}{T_{F}} H\left(t-T_{F}\right) \times \\
& \sum_{\nu=0}^{\infty}\left\{i i e r f c\left[\sqrt{\frac{R C}{4\left(t-T_{F}\right)}}(2 \nu+1-x)\right]\right\}- \\
& \left.\operatorname{iierfc}\left[\sqrt{\frac{R C}{4\left(t-T_{F}\right)}}(2 \nu+1+x)\right]\right\}
\end{aligned}
$$

where $i \operatorname{ierfc}(\xi)$ is the second repeated integral of the error function complement. The axial voltage gradient along the coil stack is given by

$$
\begin{gathered}
\frac{\partial e(x, t)}{\partial x}=2 E \frac{t}{T_{F}} \sqrt{\frac{R C}{t} \times} \\
\sum_{\nu=0}^{\infty}\left\{i e r f c\left[\sqrt{\frac{R C}{4 t}}(2 \nu+1-x)\right]+\right. \\
\quad i e r f c\left[\sqrt{\left.\left.\frac{R C}{4 t}(2 \nu+1+x)\right]\right\}-}\right. \\
\left.2 E \frac{t-T_{F} \sqrt{\frac{R C}{t-T_{F}}} H\left(t-T_{F}\right) \times}{T_{F}}(2 \nu+1-x)\right]+ \\
\sum_{\nu=0}^{\infty}\left\{\begin{array}{l}
i e r f c\left[\sqrt{\frac{R C}{4\left(t-T_{F}\right)}}(2 \nu)\right. \\
\left.i e r f c\left[\sqrt{\frac{R C}{4\left(t-T_{F}\right)}}(2 \nu+1+x)\right]\right\}
\end{array}\right.
\end{gathered}
$$

in which $\operatorname{ierfc}(\xi)$ is the first repeated integral of $\operatorname{erf} c(\xi)$. The voltage gradient is maximum at the line end, i.e., for $x=1$,

$$
\begin{aligned}
&\left|\frac{\partial e(x, t)}{\partial x}\right|_{x=1}=2 E \frac{t}{T_{F}} \sqrt{\frac{R C}{t}} \times \\
&\left\{\frac{1}{\sqrt{\pi}}+2 \sum_{\nu=1}^{\infty} i e r f c\left[\sqrt{\left.\left.\frac{R C}{t} \nu\right]\right\}-}\right.\right. \\
& 2 E \frac{t-T_{F}}{T_{F}} \sqrt{\frac{R C}{t-T_{F}}} H\left(t-T_{F}\right) \times \\
&\left\{\frac{1}{\sqrt{ } \pi}+2 \sum_{\nu=1}^{\infty} i e r f c\left[\sqrt{\frac{R C}{t-T_{F}}} \nu\right]\right\}
\end{aligned}
$$

The error function complement and its repeated integrals are defined by

$\operatorname{erfc}(\xi)=\frac{2}{\sqrt{\pi}} \int_{\xi}^{\infty} \exp \left(-\mu^{2}\right) d u$

$\operatorname{ierfc}(\xi)=\boldsymbol{\int}_{\xi}^{\infty} \operatorname{erfc}(\mu) d u$

$\operatorname{iierf} c(\xi)=\int_{\xi}^{\infty} i \operatorname{erfc} c(u) d u$

$\operatorname{ierfc}(\xi)$ and $i \operatorname{ierfc}(\xi)$ converge very rapidly towards zero for increasing values of $\xi$ and for our purpose may be taken as zero for $\xi$ greater than two. Further details can be found in the paper by Hartree $^{10}$ from which the values in the table are quoted.

It is seen from equation 21 that the voltage gradient has its maximum value for $t=T_{F}$. Let $\alpha\left(T_{F}\right)$ denote the ratio between the actual voltage gradient at the line end at $t=T_{F}$ and the average axial gradient along the coil stack, then

$$
\begin{aligned}
\alpha\left(T_{F}\right)=2 \sqrt{\frac{R C}{T_{F}}} & \left\{\frac{1}{\sqrt{ } \pi}+\right. \\
2 & \left.\sum_{\nu=1}^{\infty} i e r f c\left[\sqrt{\frac{R C}{T_{F}}} \nu\right]\right\}
\end{aligned}
$$

The contribution from the infinite series will normally be negligible, and we get 


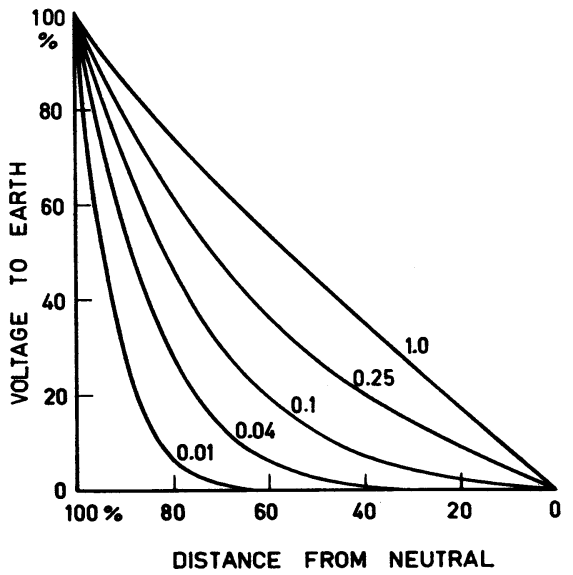

Fig. 4. Voltage distributions in interleaved windings at $t=T_{F}$ for various values of $\tau=T_{F} / R C$

the following approximate expression for the maximum relative axial voltage gradient:

$\alpha\left(T_{F}\right)=\sqrt{\frac{8 N Z C}{\pi T_{F}}}$

This equation may be written as

$\alpha\left(T_{F}\right)=\sqrt{\frac{2 T}{\pi T_{F}}} \sqrt{\frac{4}{\pi} \cdot \frac{1}{\epsilon \epsilon_{o}} \cdot \frac{N}{n p-2} \cdot \frac{1}{D} \cdot \frac{d}{b} \cdot C}$

and has a striking resemblance to equation 3 which gives the maximum relative voltage gradient at the line end as calculated for a rectangular wave from the high series capacitance hypothesis. For $T_{F}=T$ the two formulas agree within $20 \%$. For an interleaved double-section coil 5 feet in diameter containing 24 electrical turns, $T$ will be about $0.45 \mu \mathrm{sec}$ (microsecond). For this front time equation 27 gives a maximum axial gradient which is $20 \%$ less than that calculated from the high series capacitance hypothesis. If the front time is reduced to $0.2 \mu \mathrm{sec}$ the voltage gradient will be about $20 \%$ higher, and for a $0.1-\mu \mathrm{sec}$ front about $70 \%$ higher than that calculated from the high series capacitance theory. It will, however, be seen from equation 21 that these high stresses will be relaxed extremely rapidly.

The voltage distribution at $t=T_{F}$ represents the maximum axial stresses

$$
\begin{aligned}
e\left(x, T_{F}\right)= & 4 E \times \\
& \sum_{\nu=0}^{\infty}\left\{i i e r f c\left[\sqrt{\frac{R C}{4 T_{F}}}(2 \nu+1-x)\right]-\right. \\
& \left.i \operatorname{iierfc}\left[\sqrt{\frac{R C}{4 T_{F}}}(2 \nu+1+x)\right]\right\}
\end{aligned}
$$

This distribution is shown in Fig. 4 for various values of the parameter $\tau=T_{F}$ /
$R C$. The axial voltage distribution which forms the boundary condition for the train of transients which leads to the final distribution is that given by equation 19 for $t=T$. It will be still more uniform than that for $t=T_{F}$.

The voltage distributions calculated from the theory outlined here do not differ much from those calculated from the formulas derived from the high series capacitance hypothesis, except for waves with a very steep front. As it is known that the high series capacitance theory normally gives results which are reasonably close to those measured on interleaved transformers, this must be equally true for the theory proposed here. The high stresses predicted by the present theory for very steep wave fronts are relaxed so rapidly that they may easily be left unnoticed in recurrent surge oscillograph measurements. It is inherent in the theory that it will be most difficult to observe these increased stresses in windings of relatively small geometrical dimensions such as are often used in laboratory investigations.

The equations derived above do not hold for $T_{F}=0$ because the effect of the series capacitances has been neglected. This is permissible for a finite front time. For a rectangular wave, however, the series capacitances will, under quasi-stationary conditions, instantaneously carry an infinite current and the initial electrostatic voltage distribution is given by equation 1 in which the series capacitance is the same as that for a similar noninterleaved winding. This initial electrostatic voltage distribution will, however, be smoothed out extremely rapidly by the mechanism previously described.

\section{The Surge Capacitance of an Interleaved Winding}

It is a necessary consequence of the high series capacitance hypothesis that an interleaved winding must have a very high effective surge capacitance. If the series capacitance is increased about 100 times by the effect of interleaving, as claimed by Price, ${ }^{6}$ the surge capacitance should be increased by a factor of about 10 . The current flowing into the winding during the interval from $t=0$ to $t=T_{F}$ for the idealized wave of Fig. 3 would, according to the high series capacitance theory, be constant and equal to

$i_{c}=\frac{E}{T_{F}} C_{s}$

where $C_{S}$ is the surge capacitance of the winding, which should be approximately
Table I. Table of the First and the Second Repeated Integral of the Error Function

\begin{tabular}{|c|c|c|}
\hline$\xi$ & $2 i e r f c(\xi)$ & 4iierfc $(\xi)$ \\
\hline 0.00 . & 1.1284 & .1 .0000 \\
\hline 0.05 . & .1 .0312 . & .0 .8921 \\
\hline 0.10 . & 0.9396 & . .0.7936 \\
\hline 0.15 . & 0.8537 & .0 .7040 \\
\hline 0.20 . & 0.7732 & 0.6227 \\
\hline 0.25 . & 0.6982 & $\ldots 0.5491$ \\
\hline 0.30 . & . .0.6284 & .0 .4828 \\
\hline 0.35 . & 0.5639 & $\ldots 0.4233$ \\
\hline 0.40 . & 0.5043 & . 0.3699 \\
\hline 0.45 . & 0.4495 & . .0.3223 \\
\hline 0.50 . & 0.3993 & . .0.2799 \\
\hline 0.55 . & 0.3535 & . 0.2423 \\
\hline 0.60 . & .0 .3119 & $\ldots 0.2090$ \\
\hline 0.65 . & . 0.2742 & $\ldots 0.1798$ \\
\hline 0.70 . & ..0.2402 & $\ldots 0.1541$ \\
\hline 0.75 . & . .0.2097. & $\ldots 0.1316$ \\
\hline 0.80 . & 0.1823 & $\ldots 0.1120$ \\
\hline 0.85 . & .0 .1580 & $\ldots 0.0950$ \\
\hline 0.90 . & .0 .1364 & $\ldots 0.0803$ \\
\hline 0.95 . & 0.1173 & $\ldots 0.0677$ \\
\hline 1.00 . & 0.1005 & $\ldots 0.0568$ \\
\hline 1.10 . & .0 .0729 & $\ldots 0.0396$ \\
\hline 1.20 . & $\ldots 0.0521$ & $\ldots 0.0272$ \\
\hline 1.30 . & $\ldots 0.0366$ & $\ldots 0.0184$ \\
\hline 1.40 . & $\ldots 0.0253$ & $\ldots 0.0122$ \\
\hline 1.50 . & 0.0172 & $\ldots 0.0080$ \\
\hline 1.60. & 0.0115 & $\ldots 0.0052$ \\
\hline 1.70 . & 0.0076 & $\ldots 0.0033$ \\
\hline 1.80 . & .0 .0049 & $\ldots 0.0021$ \\
\hline 1.90 . & ..0.0031 & $\ldots 0.0013$ \\
\hline 2.00 & $\ldots 0.0020$ & $\ldots 0.0008$ \\
\hline 2.10 . & ..0.0012. & $\ldots 0.0005$ \\
\hline 2.20 . & . 0.0007 & $\ldots 0.0003$ \\
\hline 2.30 . & ..0.0004 & $\ldots 0.0002$ \\
\hline 2.40 . & .0 .0002 & $\ldots 0.0001$ \\
\hline 2.50 . & .0 .0001 & . .0.0000 \\
\hline
\end{tabular}
Complement

The Functions 2 ierfc $(\xi)$ and 4 iierfc $(\xi)$

$C_{s}=\sqrt{\frac{\pi}{4} \cdot \epsilon \epsilon_{0} \cdot \frac{n p-2}{N} \cdot D \cdot \frac{b}{d} \cdot C}$

It is clear from the mode of operation proposed in this paper that the concept of a surge capacitance cannot be applied to an interleaved winding except in relation to a true rectangular wave. For such a wave the winding will initially at $t=0$ behave like a surge capacitance which, however, will be precisely the same as that for a similar conventional disk winding with the same physical dimensions. The current flowing into an interleaved winding during the initial period is

$i(t)=\frac{1}{R}\left|\frac{\partial e(x, t)}{\partial x}\right|_{x=1}$

which reaches its maximum value $i\left(T_{F}\right)$ at $t=T_{F}$

$i\left(T_{F}\right)=\frac{E}{T_{F}} \sqrt{2 \cdot \epsilon \epsilon_{0} \frac{n p-2}{N} \cdot D \cdot \frac{b}{d} \cdot C \frac{T}{T_{F}}}$

This current is very much higher than it would be had the winding not been interleaved. It is of the same order of magnitude as that predicted from the high series capacitance hypothesis. This explains why an interleaved winding superficially will appear to have a relative high surge capacitance. 


\section{Conclusions}

The high series capacitance theory for the impulse behavior of interleaved windings is based on the assumption that interleaving of turns which are electrically about half a coil apart results in a large increase in the electrostatic series capacitance. This is, however, logically incompatible with the idea of an initial electrostatic voltage distribution. As far as capacitances are concerned, the effect of interleaving must be restricted to such cases where currents are actually flowing through the turns. This is the case for the transients leading from the initial to the final distribution, and it explains the pronounced smoothing effect of interleaving on these transients.

The interleaving of the turns results in the formation of surge impedances through which a high current flows into the winding during an initial period thereby charging up the distributed earth capacitances. This leads to a voltage distribution which is much more uniform than it would be for a similar noninterleaved winding. The conclusions which can be drawn from this hypothesis differ in some important ways from those derived from the high series capacitance hypothesis. For front times shorter than the duration of the initial period the maximum axial voltage gradient will increase inversely proportional to the square root of the front time. For extremely short front times or for steeply chopped waves the axial voltage gradients will be as high as for a similar conventional disk-type winding. These high stresses will, however, be relaxed very rapidly. Considering the risk for failures due consideration must, therefore, be given to the short-time impulse breakdown characteristics of the insulation, and a design based on the high series capacitance hypothesis will probably not involve any risks.

Another important difference is related to the maximum interturn voltages. These will, according to the high series capacitance theory, be equal to half the initial voltage across a double section coil. According to the theory proposed in this paper the interturn voltages will have a tendency to oscillate if the rise time of the voltage across a coil is shorter than the duration of the initial period. This will, ignoring the attenuation, lead to a doubling of the interturn voltages. These oscillations should be more pronounced in coils with a large number of electrical turns per disk.

In order to achieve the best possible response to surge voltages an interleaved transformer winding should be designed so that the initial period $T$ is as small as possible and the ratio between $T$ and $2 N Z C$ as large as possible. $T$ and $Z$ are given by equations 8 and $9, N$ is the number of double-section coils, and $C$ is the total capacitance to earth.

The theory outlined here is, necessarily, very approximate, and many of the assumptions made are, rigorously speaking, not true. Similar limitations do, however, also hold for the high series capacitance theory. It has merely been the intention to show that the effect of interleaving the electrical turns of a transformer winding on the response of the winding to surge voltages can be explained without recourse to the concept of a high series capacitance.

\section{References}

1. A New Type of Transformer Winding Giving ImProved ImpUlse Voltage DistribuTION, A. T. Chadwick, J. M. Ferguson, D. H. Ryder, G. F. Stearn. Report no. 107, CIGRE, Paris, France, 1950.

2. Design of Power Transformers to WithStand Surges Due to Lightning, with Special REFERENCE TO A New TYPe OF Winding, A. T. Chadwick, J. M. Ferguson, D. H. Ryder, G. F. Stearn. Proceedings, Institution of Electrical Engineers, London, England, vol. 97, pt. II, 1950 , p. 737.

3. IMPROVED CORE Form TRANSFormer Winding, E. J. Grimmer, W. L. Teague. AIEE Transactions, vol. 70 , pt. I, 1951, pp. 962-67.

4. L'AVVOLGIMENTO CON BOBINE A SPIRE INTERCALATE NEI TRASFORMATORI AD ALTA TENSIONE M. Ferrari Bardile. Rendiconti della $58^{\mathrm{a}}$ riunione annuale dell' Associazione Elettrotecnica Italiana, Rome, Italy, 1957, paper no. 519.

5. Stossspannungssichere TransformatorwickLUNGEN, H. Brechna. Bulletin Oerlikon, Zürich, Switzerland, no. $328 / 329,1958$, p. 89.

6. Interleaved Transformer Windings, J. B. Price. Electrical Review, London, England, vol. 165,1959 , p. 927

7. Impulse Stresses in Transformer Windings R. A. Zambardino. Electrical Times, London, England, vol. 137, 1960, pp. 3, 81.

8. LA SOllecitazione A impulso NEI tRasFORMATORI CON AVVOLGIMENTI A SPIRE INTERCalate, V. Gorio. L'Elettrotecnica, vol. 47, 1960, p. 78 .

9. Bestimmung Der Stossspannungsverteilung IN Transformatoren MIT Digitalrechner, Bangalore Narayanamurti Jayaram. ETZ-A, Berlin, Germany, vol. 82, 1961, p. 1.

10. Some Properties and Applications of the REPEATED INTEgRals OF THE ERROR FunCtion, D. R. Hartree. Memoirs and Proceedings of the Manchester Literary and Philosophical Society Manchester, England, vol. 80, 1936, p. 85.

\section{Discussion}

R. A. Zambardino (English Electric Company Ltd., London, England): The discusser has found the paper presented by Mr. Pedersen extremely interesting. Many points in his theoretical approach and in his mathematical treatment of the interleaved winding will be a valuable contribution to any future complete study of the impulse behavior of transformer windings.

However, my company, as originator and most experienced user of the interleaved winding, has comments and reservations to make regarding the premises and conclusions of this study.

\section{Comparison of THE SURge-IMPedance} and Series Capacitance Theories

The first important point is to clarify the relationship between the surge-impedance approach presented in this paper in regard to the series capacitance theory.

The paper might give the impression, see the Summary, that the series capacitance theory is to be criticized and is in conflict with the new method discussed therein.

It should, in fact, be noted that the value of capacitance assumed by the author for his method of calculation is absolutely identical to that of the series capacitance theory (see equation 4) which gives the capacitance per section in his theory, and this is exactly $2 N$ times the total capacitance in the series capacitance theory as given in equation 2 .

The author is simply noting that this capacitance, which value he fully accepts, cannot be charged at once but has a finite charging time, determined mainly by its charging inductance, for which formulas are given (see equations 6 and 9 ). This is, of course, true, not only for the interleaved winding but for any winding and, indeed, for any capacitance whatsoever.

Once the problem is seen in this light the following consequences will logically follow:

1. This series capacitance theory is perfectly valid whenever the charging time is small compared with the front time of the applied wave. This, we can assume, is agreed to by the author considering his statements in the section entitled "The
Initial Voltage Distribution in Interleaved Windings," and in the "Conclusions." The striking resemblance of the gradient's equations 7 and 3 is in fact perfectly natural, since both are derived from identical values of capacitance.

2. The new method presented is not an alternative to the series capacitance theory, but an attempt to extend its application to applied waves with front times which are short in comparison with the charging time of the series capacitances.

3. The basic approach presented in this paper is of more general application than to the interleaved windings only. The principles expounded are relevant to the theoretical study of transformer windings in general.

4. The introduction of the charging inductance associated with the series capacitance of the interleaved winding emphasizes what is in fact a major advantage of these windings, namely, their extremely short charging time. The fact that the charging inductance is that of the two strands of interleaved conductors makes this parameter very small, and ensures that the charging 


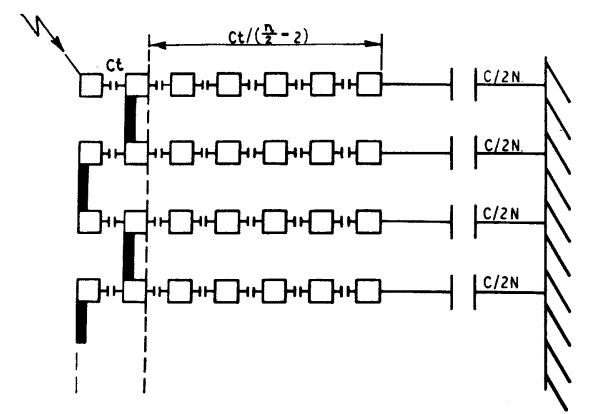

Fig. 5. Equivalent circuit

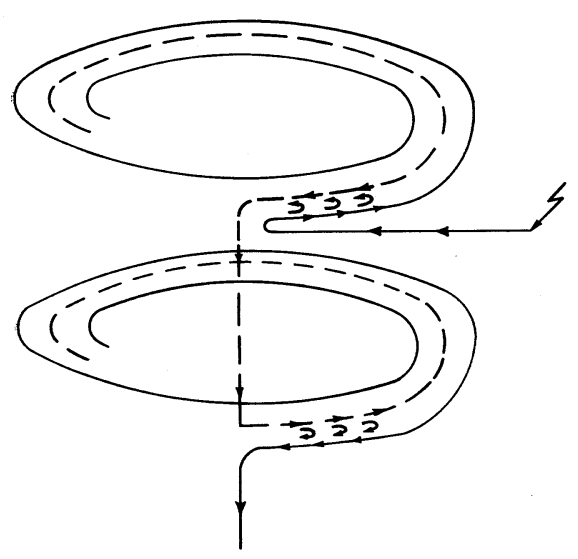

Fig. 6. Current path and equivalent circuit of an interleaved winding for hypothetical waves with fronts of a few millimicroseconds

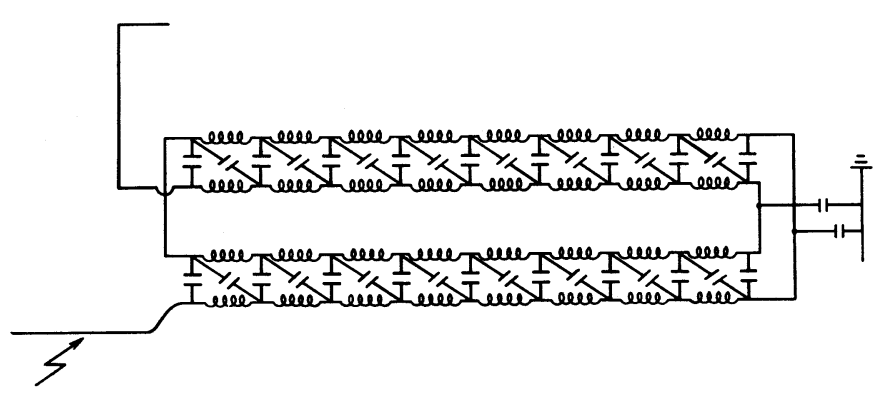

Fig. 7. Equivalent circuit of two inter-

leaved sections

APPLIED VOLTAGE

VOLTAGE ACROSS LINE END COIL
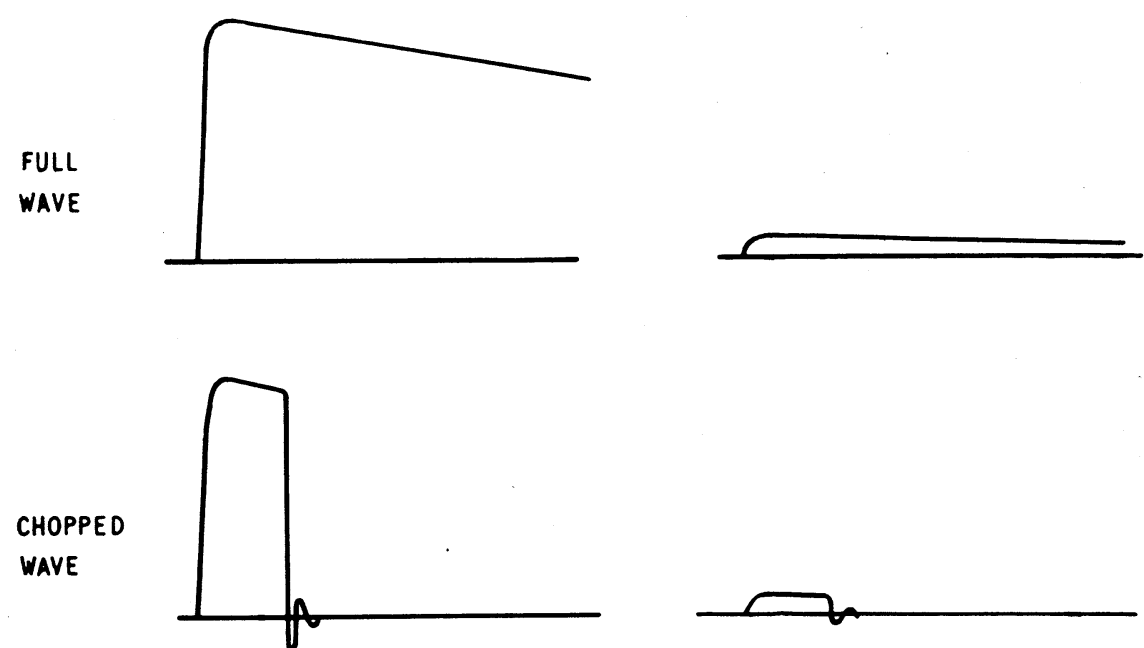

Fig. 8. Applied voltage and voltage across an interleaved line end coil produced by the front of the wave and not during the chopping. The gradients produced by the chopping subtract from those already established by the front of the incoming wave (see Fig. 5). Even if for some special transformer the chopping were so sharp as to be faster than the charging time of the coil, this would at least need to double the gradient calculated with the series capacitance theory before the over-all actual stress, i.e., the difference between the gradient due to the front and that due to the chopping, became greater than that produced by the front of the wave. An increase by this amount is out of the question, even on the basis of the theory produced in this paper (see equation 27 giving an increase in stress of $70 \%$ for a $0.1-\mu \mathrm{sec}$ front). Therefore, the maximum stress during chopped waves is produced by the front, i.e., before chopping, for which the series capacitance theory is fully valid.

\section{Behavior of Interleaved CoIls}

Under Hypothetical Surges Having

Extremely Short Fronts, of The

Order of a Few Millimicroseconds

We have already discussed the principles on which is based the theory developed in the paper. This has shown that they are applicable to surges having a front much faster than could be produced in practice under test or service conditions. Such hypothetical conditions are, however, of theoretical interest and we would now like to make some comments on the method of calculation developed in the paper. We foundr ather baffling the statement that "for extremely short front time, . . ., the axial voltage gradients will be as high as for a conventional disk-type winding" (see "Conclusions"). Since the author has noted that it takes only a few millimicroseconds for an applied wave to travel along the interleaving joints at the outside of the coil stack, the statement quoted is obviously untenable, even for completely imaginary wave with fronts of a few millimicroseconds. For such rather metaphysical waves the capacitive circuit of the interleaved would be as in Fig. 6 . The series capacitance would be that given by the first pair of interleaved turns (for $p=1$ ) and the ground capacitance $C$, being generally at the inside of the coil, would be reduced by being in series with the remaining turn capacitances in series with each other. The same result could be obtained in a much more complicated way, using the surge-impedance theory, but taking into account the full equivalent circuit of the coil as shown in Fig. 7. The fact that the surge may not have traveled round a full turn is immaterial, since this would vary by the same amount both series and ground capacitance. If the original $\alpha$ (calculated with the full series capacitance of the coil) is between 1 and 3 the $\alpha^{x}$ calculated with the circuit of Fig. 6 would be approximately between 3 and 6 , respectively.

The voltage distribution given by the $\alpha^{x}$ is the worst which could be produced by imaginary fronts of a few millimicroseconds.

Formulas derived from the surge impedance theory should give this distribution as a limit for $T_{f}$ tending to zero (or, to be more precise, $T_{f}$ tending to 1 or 2 millimicro- 
seconds). The voltage distributions derived in the paper (shown in Fig. 4) go well below the above distribution and, therefore, the formulas given in the paper must be considered as unduly pessimistic, even for the hypothetical range of front times for which they could apply. The circuit shown in Fig. 2 of the paper should be modified so that for $T_{f}$ tending to practically zero (a few millimicroseconds) it should reduce to a circuit equivalent to that shown in our Fig. 6 . If this is done the method of calculation based on surge impedances should give, even for the hypothetical conditions where it applies, results considerably nearer to those derived from the series capacitance theory.

The approach to the calculation of surge voltage distribution, presented by $\mathrm{Mr}$. Pedersen, is of great theoretical interest and its principles could usefully be extended to the study of transformer windings of any type.

Its application to interleaved windings is limited by the fact that the range of applied wave front times to which it would apply is in practice below the range of front times which occur in service or test conditions. For the latter front times the series capacitance theory hitherto used is fully valid.

For the hypothetical very-steep-fronted waves to which the theory would apply, the results derived in the paper are unduly pessimistic, as the equivalent circuit used neglects elements which play an important part. If it were objected that, strictly speaking, a $T_{f}$ of 1 millimicrosecond is still not zero, it should be noted that, apart from the physical impossibility of getting a zero rise time, all the impulse calculation theories would break down for such conditions and all windings, however built, would look the same. If the rise time is zero the wave reaches the winding before any current has flown through it. The field distribution at $t=0$ is identical with that which would be obtained if a horizontal electrode (the bushing lead) was brought up to but not connected to the winding. The field conditions would be 3 -dimensional with a concentrated field radiating from the point where the bushing lead enters the winding. The actual characteristics of any winding would be immaterial in such conditions, which are entirely a figment of the imagination.

G. M. Stein and J. M. McWhirter (Westinghouse Electric Corporation, Sharon, $\mathrm{Pa}$ ): The author's contention that the interleaved winding has not a higher series capacitance than the ordinary disk winding cannot be accepted in a practical sense for the following reasons:

The author postulates that, for establishing a pure electrostatic voltage distribution, no current is allowed to flow through inductances, that is, in this case in the conductors of a transformer winding. Since the cross section of the wires supplying voltage to each end of the coil is usually very small and their distance very large, as compared with the spacing and size of the capacitor surfaces formed by the conductors, the series capacitances accounting for that pure electrostatic voltage distribution are practically zero.

In order to create the field and to justify the use of the series capacitances appearing in conventional calculations of the initial, that is, short-time, surge distribution in an ordinary disk winding, current must pass at least through the turn adjacent to each line terminal and through any static plates used, that is, current must flow in conductors with inductances. After charging the static plate and the first conductor, this current seems to prefer the shorter and less inductive path through the dielectric between turns and between coils to the high inductive path through the other conductors, because such a flow places the capacitances between all conductors in series and surge calculations based on this condition are borne out by test results.

In an interleaved winding, however, distant conductors are connected together so that the current, after passing through the first turn seems to prefer the path through the other conductors to the path through the dielectrics between them since this flow charges the capacitances between adjacent conductors of an interleaved group in parallel, and calculations of the initial surge distribution made under this condition agree with test results. It is this paralleling effect which permits the author to treat the winding like a transmission line and thus, to introduce the time element into the analysis, because the capacitances of such a line appear parallel connected in its wave resistance. If one quarter period $T$ of the oscillation occurring during the charging period becomes longer than the time $T_{F}$ to maximum peak in the applied voltage wave, he finds the paralleling effect on the series capacitances reduced to the extent that these capacitances tend to be the same in ordinary and interleaved disk windings for sufficiently short rise times $T_{F}$.

To summarize, a conductor current has to flow only in the turn adjacent to each line terminal in order to explain the series capacitance in ordinary disk windings while current has to pass through the whole winding for obtaining the series capacitance corresponding to surge phenomena observed when the coils are interleaved. Consequently, inductive currents have to be present in both cases which are distinguished from each other only by the extent of this current flow.

By introducing this inductive effect, and thus, the time element, into the analysis of interleaved disk coils, the present paper becomes a major contribution to our knowledge of the functioning of this type of winding.

A. Pedersen: I am very grateful to $\mathrm{Mr}$. Zambardino, and to $\mathrm{Mr}$. Stein and $\mathrm{Mr}$. McWhirter, for their discussions which clarify some problems which I have dealt with too superficially.

The concepts of series and earth capacitance originate from studies of the impulse response of a uniformly wound single-layer helical coil for which these capacitances have a simple geometrical interpretation. In conventional calculations of voltage distributions in actual transformer windings the formulas derived for the simple helical coil are used without modifications. To do this, equivalent series and earth capacitances must be introduced. In the published versions of the higher series capacitance theory the equivalent series capacitance is found by considering the electrostatic energy in the interturn insulation. To find this energy a linear voltage distribution along the electrical turns is assumed (a distribution which, by the way, cannot be achieved electrostatically). In this way equation 2 of the paper is derived. Mr. Zambardino points out that this is the same capacitance per coil as that involved in the surge capacitances. This is, however, entirely accidental because equation 2 depends on an arbitrarily chosen voltage distribution within a coil.

I disagree with Mr. Zambardino's statement that "the series capacitance theory is perfectly valid whenever the charging time is small compared with the front time of the applied wave." In this case the mutual and self-inductances must be taken into account, and the distribution is thus not even approximately electrostatic, and the use of equation 1 is, consequently, meaningless. For such slow wave fronts interleaving will, as stated in the paper, greatly influence the capacitance of the winding. This is, however, inherently associated with currents flowing through the main inductances. The difference between the ordinary disk winding and the interleaved winding under such conditions will be as described by $\mathrm{Mr}$. Stein and Mr. McWhirter. But the high capacitance thus achieved by interleaving is not the electrostatic series capacitance involved in equation 1 . And the fact that this equation, when applied to cases for which it does not hold, appears to give reasonable results if one of its fundamental parameters is replaced by a quantity which is derived from an entirely different situation as that described by equation 1 , can only emphasize that the high series capacitance theory is based on assumptions which are fundamentally wrong.

I do, of course, agree with Mr. Zambardino that a strictly rectangular wave is a hypothetical condition, but I think that he is unduly optimistic in asserting that the finite charging time of the transmission lines formed by the coils will, in all practicalcases, be small compared with the front times which could occur in service or test conditions. With data quoted from reference 1 , the transit time is half a $\mu \mathrm{sec}$, which certainly is not small compared to what could reasonably be expected during service conditions.

Concerning the distribution for very steep fronted waves, Mr. Zambardino views the problem qualitatively in the same way as I do (see section $B$ in Fig. 6). However, when it comes to the quantitative estimation of the distribution, Mr. Zambardino is wrong in assuming that the equivalent circuit is that shown in Fig. 6 for the following reason. The current flowing into the "series capacitance" formed by the first pair of interleaved turns will be strictly proportional to the voltage applied across this pair of turns (it is a current through a surge impedance) and not to the derivative of this voltage with respect to the time, i.e., the differential equation for the distribution is equation 10 of the paper and not

$$
\frac{d^{2} e(x)}{d x^{2}}=\alpha^{2} e(x)
$$

as assumed by Mr. Zambardino. For a front time of 10 to 15 millimicroseconds and a transit time of $0.5 \mu \mathrm{sec}$, equation 10 will give a maximum axial stress which is approximately 4 to 5 times the stress predicted from 
the conventional high series capacitance theory; see equation 27. The apparent value of $\alpha$ would thus approach 15 and not 6 as stated by Mr. Zambardino, i.e., the distribution would approach the pure electrostatic distribution for a rectangular wave. I cannot, therefore, accept Mr. Zambardino's statement that the results derived in the paper are unduly pessimistic. I would, however, like to emphasize that both the theory in the paper and the high series capacitance theory imply quasi-stationary conditions, and this will not be the case for extremely short front times. In such cases the circuit theoretical approach should be replaced by field theoretical considerations.

In conclusion $\mathrm{I}$ wish to thank $\mathrm{Mr}$. Zambardino and Mr. Stein and Mr. McWhirter for their valuable and stimulating discussions of my paper.

\title{
Compressed Gas Insulation in the Million-Volt Range: A Comparison of $\mathrm{SF}_{6}$ with $\mathrm{N}_{2}$ and $\mathrm{CO}_{2}$
}

\author{
S. F. PHILP \\ SENIOR MEMBER IEEE
}

\begin{abstract}
Summary: Maximum voltage which can be insulated between a sphere and a plane has been measured as a function of gas pressure and gap. It is found to be approximately three times higher in $\mathrm{SF}_{6}$ than in $\mathrm{N}_{2}+\mathrm{CO}_{2}$, up to pressures of roughly $9 \mathrm{~atm}$ (atmosphere). For higher pressures the relative superiority of $\mathrm{SF}_{6}$ over $\mathrm{N}_{2}+\mathrm{CO}_{2}$ diminishes. Gradients of more than 100 Mv (million volts) per $\mathrm{m}$ (meter) were insulated on a $19-\mathrm{mm}$ (millimeter)-diameter electrode in $20 \mathrm{~atm}$ of $\mathrm{SF}_{6}$.
\end{abstract}

$\mathbf{T}$ E maximum voltage which can be insulated between a sphere and a plane has been measured as a function of gas pressure and gap. The objective has been particularly to compare the insulating ability of sulfur hexafluoride with that of a mixture of equal parts of $\mathrm{N}_{2}$ and $\mathrm{CO}_{2}$, a commonly used insulating gas mixture. It is found that the maximum voltage is approximately three times higher in $\mathrm{SF}_{6}$ than in $\mathrm{N}_{2}+\mathrm{CO}_{2}$, up to pressures of roughly $9 \mathrm{~atm}$. For higher pressures the relative superiority of $\mathrm{SF}_{6}$ over $\mathrm{N}_{2}+\mathrm{CO}_{2}$ diminishes. Gradients of more than $100 \mathrm{Mv}$ per $\mathrm{m}$ were insulated on s. 19 -mm-diameter electrode in 20 atm of $\mathrm{SF}_{6}$.

Gases whose molecular structure in-

Paper 63-27, recommended by the AIEE Electrical Insulation Committee and approved by the AIEF Technical Operations Department for presentation at the IEEE Winter Ceneral Meeting New York at the IEEE Winter General Meeting, New York, N. Y., January 27-February 1, 1963. Manuscrip submitted May 1, 1961; made available for printing October 23, 1962.

S. F. PHILP is with the Massachusetts Institute of Technology, Cambridge, Mass.

The author wishes to express his appreciation to Professor John G. Trump for his help and guidance. It is a pleasure to acknowledge also the support of the National Science Foundation. volves a large number of atoms often have good insulating properties. $^{1-4}$ In addition, many of the heavy gases will attach electrons to form negative ions, a process which augments their insulating properties. ${ }^{5,7,8}$

To be practically useful for insulating purposes, these heavy gases should:

1. Be chemically inert, at least to the extent that they will not attack plastics, metals, and other substances which are commonly used in high-voltage equipment.

2. Be readily available and not too expensive.

3. For very-high-voltage applications it is sometimes desirable that the gas have sufficiently high vapor pressure to be used at pressures of $10 \mathrm{~atm}$ or more at ordinary temperatures.

Sulfur hexafluoride $\left(\mathrm{SF}_{6}\right)$ is an outstanding example of a gas which fulfills these requirements and has particularly good insulating properties. $\mathrm{SF}_{6}$ is very inert and satisfies the first requirement; however, it should be noted that the molecular fractionsformed during the passage of an electric discharge are corrosive and highly toxic. ${ }^{9}$ The high vapor pressure of $\mathrm{SF}_{6}$ (24 atm at standard temperature) makes it of greater interest for insulation of voltages in the million-volt range than gases such as carbon tetrachloride or freon whose vapor pressures are 0.13 atm and $5 \mathrm{~atm}$, respectively. However, a mixture of these heavy gases with a lighter gas such as nitrogen or hydrogen results in an increase of insulating strength for the same total gas pressure, particularly for small amounts of added carbon tetrachloride or freon. ${ }^{3,4,10,11,24}$
The measurements reported here are concerned only with a comparison between pure $\mathrm{SF}_{6}$ and a gas consisting of equal parts of $\mathrm{N}_{2}$ and $\mathrm{CO}_{2}$. This mixture was chosen for comparison because it is commonly used in insulation, having about the same insulating properties as compressed air without the concomitant combustion hazard.

\section{Apparatus}

The voltage source used in these experiments is a Van de Graaff electrostatic generator. This machine, identical to one described in detail elsewhere, ${ }^{12}$ has an insulating column 24 inches long and 15 inches in diameter. The high-voltage terminal is a polished stainless-steel spinning also 15 inches in diameter and has the form of a cylinder surmounted by a hemisphere. The generator is enclosed in a cylindrical pressure vessel of 30 -inch diameter.

The test gas was in all cases technical grade gas.

The experiments were performed within the pressure vessel of the generator. The test electrodes, polished steel ball bearings, were introduced through a port in the vessel and supported on metal rods which could be moved from outside. The geometry is approximately that of a sphere facing an infinite plane, since the high-voltage terminal is large compared to either the gap or the spherical electrode.

A generating voltmeter is used to measure the terminal potential. This voltmeter is in principle a linear device, and it is found in practice to be very accurately linear except for a small region near zero voltage. ${ }^{13}$ The voltmeter is calibrated for terminal potentials up to $80 \mathrm{kv}$ by introducing a potential, measured with a laboratory standard highvoltage resistor, from an external $\mathrm{d}-\mathrm{c}$ power supply. In addition, a calibration point in the $\mathrm{Mv}$ range is obtained by using the bremsstrahlung produced by electrons accelerated in the tube of the Van de Graaff generator, in an experimental determination of the threshold for a gamma-ray-induced nuclear reaction. ${ }^{14}$ This calibration was completed before 